Article

\title{
Photocatalytic Properties of Nb/MCM-41 Molecular Sieves: Effect of the Synthesis Conditions
}

\section{Caterine Daza Gomez * and J. E. Rodriguez-Paez}

Science and Technology in Ceramic Materials Group (CYTEMAC), Department of Physics_FACNED, University of Cauca, Popayan 190001, Colombia; E-Mail: jnpaez@unicauca.edu.co

* Author to whom correspondence should be addressed; E-Mail: katerine-16-@hotmail.com.

Academic Editor: Anibal Maury-Ramirez

Received: 1 April 2015 / Accepted: 13 August 2015 / Published: 20 August 2015

\begin{abstract}
The effect of synthesis conditions and niobium incorporation levels on the photocatalytic properties of $\mathrm{Nb} / \mathrm{MCM}-41$ molecular sieves was assessed. Niobium pentoxide supported on MCM-41 mesoporous silica was obtained using two methods: sol-gel and incipient impregnation, in each case also varying the percentage of niobium incorporation. The synthesized $\mathrm{Nb}-\mathrm{MCM}-41$ ceramic powders were characterized using the spectroscopic techniques of infrared spectroscopy (IR), Raman spectroscopy, X-ray diffraction (XRD), and transmission electron microscopy (TEM). The photodegradation capacity of the powders was studied using the organic molecule, methylene blue. The effect of both the method of synthesis and the percentage of niobium present in the sample on the photodegradation action of the solids was determined. The mesoporous Nb-MCM-41 that produced the greatest photodegradation response was obtained using the sol-gel method and 20\% niobium incorporation.
\end{abstract}

Keywords: synthesis; mesoporous; niobium; photodegradation

\section{Introduction}

A photocatalyst is a material able to absorb light efficiently and thereby induce a chemical reaction [1]. In the 1970s, the photo-response of solid materials became a very important research topic due to the potential applications of this phenomenon in new technologies. This led to heterogeneous photocatalysis encouraging unique developments with applications in alternative energy, organic synthesis and environmental treatment [2], with semiconductor compounds being most widely used for 
this purpose. Of these, $\mathrm{TiO}_{2}$ and $\mathrm{TiO}_{2}$-based materials have by far been the most attractive economically: they are authoritative photocatalysts also because they are nontoxic and offer among other advantages ready availability, chemostability, and reusability. Other inorganic semiconductors have been successfully used as photocatalysts, principally in environmental heterogeneous photocatalysis. These include CdS, $\mathrm{WO}_{3}, \mathrm{SnO}_{2}, \alpha-\mathrm{Fe}_{2} \mathrm{O}_{3}, \mathrm{AgNbO}_{3}$ and $\mathrm{SrTiO}_{3}$ [3]. Basically, when such semiconductor photocatalysts absorb light, electronic excitation occurs that can be used in chemical or electrical processes.

During all this time, knowledge in disciplines such as photochemistry, catalysis and semiconductor physics has grown and allowed a deeper understanding of the phenomenon of photocatalysis, which depends mainly on the interaction of radiation with the solid and its surface reactivity [4]. The basic concepts underlying the phenomenon of photocatalysis have been obtained from studies done on photocatalytic systems employing microcrystalline (bulk) semiconductor photocatalysts. Recently, nanotechnology has opened up exciting new possibilities to develop more efficient photocatalysts [5]. This modernization of photocatalysis and nanophotocatalysis has been encouraged by progress that has been made in the physics and chemistry of nanoscale semiconductor particles exhibiting quantum size effects, i.e., a dependence on the fundamental properties of these nanosized particles. The phenomenon of quantum confinement, which these nanoparticles exhibit, favors changes in the electronic, optical, photochemical and photocatalytic properties of nanocrystalline semiconductors [6,7].

Much work has been published in the last two decades related not only to colloidal semiconductors but to other types of nanostructure materials, including: nanocrystalline powders, coupled nanocomposites, nanocrystalline films, mesoporous semiconductors, etc. Clearly, although nanoparticle semiconductors have high surface energy compared to bulk semiconductors, they tend to reduce surface tension by aggregation. In order to prevent or delay aggregation and counter the coarsening of the nanocrystals, a stabilizer or surfactant is added during synthesis that is adsorbed on them, thereby reducing surface tension and creating a steric or electrostatic barrier. In addition, this characteristic shown by the nanoparticles can be used to create a new class of photocatalysts with a porous structure [8]. For this, surfactant type compounds are introduced during synthesis in suitable concentrations, not so much to prevent aggregates from forming but to form a nanoporous structure in the aggregates. As photocatalysts, mesoporous materials have a number of advantages, including: (1) a high value of specific surface area and nanometer pore size; (2) prolonged substrate retention; (3) migration of photogenerated charge carriers through the adjacent semiconductor nanoparticles, and an accumulation at their points of contact, and (4) high possibility of repeat light reflectance encouraging a greater absorption of light [9]. The benefits of these mesoporous structures are illustrated by the titania nanophotocatalysts in the photocatalytic oxidation of dyes [10], oxidative disruption of the cell membranes of $E$. coli bacteria [11] and carbon dioxide reduction to methanol [12], among others. Specifically, mesoporous ZnO [13], $\mathrm{CeO}_{2}$ [14] and $\mathrm{Co}_{3} \mathrm{O}_{4}$ [15] have all exhibited high photocatalytic activity in the destruction of dyes when compared with non-porous nanopowders.

Specifically, considering mesoporous niobium compounds, in one of the first works where the mesoporous niobium-containing silicate of MCM-41 was synthesized, the results of X-ray diffraction and transmission electron microscopy confirmed that this material exhibited an ordered mesoporous structure that could be altered by applying external pressure ( $\sim 50 \mathrm{MPa})$ [16]. According to these researchers, the niobium-containing MCM-41 structure is mechanically less stable than pure silica MCM-41. The mesoporous molecular sieves were then modified with ammonium and copper cations 
and tested in several catalytic reactions [17]. An important result of this study showed that mesoporous NbMCM-41 was an attractive catalyst for the selective oxidation of thioethers to sulphoxides with $\mathrm{H}_{2} \mathrm{O}_{2}$

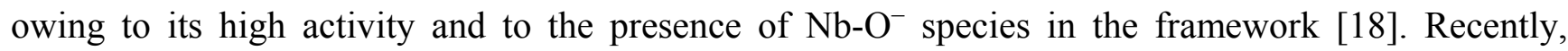
mesoporous $\mathrm{Nb}_{2} \mathrm{O}_{5}$ was used as a photocatalyst instead of the nanocrystalline niobium oxide compound, and its quantum yield of hydrogen evolution from aqueous methanol solutions increased by a factor of 20 [19]. When the mesoporous $\mathrm{Nb}_{2} \mathrm{O}_{5}$ samples were loaded with nanoparticles of $\mathrm{Pt}, \mathrm{Au}, \mathrm{Cu}$ and $\mathrm{NiO}$, their photocatalytic activity increased such that the rate of $\mathrm{H}_{2}$ evolution from an aqueous methanol solution under UV irradiation changed, being higher for the Pt-loaded niobium oxide catalysts [20]. Another important application of $\mathrm{Nb}_{2} \mathrm{O}_{5}$ photocatalysts has been the oxidation of organic compounds in aqueous media [21]. This involves natural and synthetic $\mathrm{Nb}_{2} \mathrm{O}_{5}$ being placed initially in the presence of hydrogen peroxide and methylene blue [22] and in another study niobium pentoxide was modified by doping it with molybdenum or tungsten and then treated with $\mathrm{H}_{2} \mathrm{O}_{2}$, significantly increasing the photocatalytic activity of this compound in the oxidation of methylene blue dye [23].

This article reports obtaining Nb/MCM-41 molecular sieves by using two synthesis methods: the traditional incipient impregnation method and the sol-gel process. After characterizing the synthesized powders, their photodegradation capacity was evaluated using methylene blue as a pattern compound. Finally, also with regard to their photodegradation capacity, the effect of the synthesis method for the $\mathrm{Nb} / \mathrm{MCM}-41$ photocatalysts was determined, together with that of the niobium content in the different samples.

\section{Experimental Procedure}

\subsection{Synthesis of Different Molecular Sieves}

\subsection{1. $\mathrm{Nb}_{2} \mathrm{O}_{5}$ Synthesis Supported on MCM-41 through the Sol-Gel Method (Nb-MCM-41-Solgel)}

A total of $8.8 \mathrm{~g}$ of N-cetyl-N,N,N-trimethylammonium bromide (Merck, Darmstadt, Germany) were dissolved in a solution of $208 \mathrm{~mL}$ of deionized water and $96 \mathrm{~mL}$ of aqueous ammonia solution $(25 \% \mathrm{w} / \mathrm{w}$, Merck, Darmstadt, Germany) at $35^{\circ} \mathrm{C}$. A total of $40 \mathrm{~mL}$ of TEOS (98\% purity, Aldrich ${ }^{\circledR}$, San Luis, MO, USA) and an aqueous solution of ammonium tris(oxalate) complex of niobium(V) (CBMM, Araxá, Minas Gerais, Brazil) were slowly added under agitation to this clear solution, in $0.1 \mathrm{M}$ of oxalic acid; the amounts of niobium oxalate aggregated were $0.4756,0.9512$, and $1.4268 \mathrm{~g}$ to obtain niobium percentages of $10 \%, 20 \%$, and $30 \%$, respectively, in the mesoporous silica material. Thereafter, the system was agitated for three hours and the gel aged at room temperature for $24 \mathrm{~h}$ in a sealed container. The precipitate was filtered, washed with $600 \mathrm{~mL}$ of deionized water, and air dried at room temperature. Finally, to eliminate the organic matter, it was calcined at $550{ }^{\circ} \mathrm{C}$ for eight hours. The procedure previously described was carried out taking into account its simplicity, reproducibility, and the infrastructure currently available in the laboratory. Certainly, the aim in future would be to incorporate further stages, used to good effect by other researchers, in order to optimize the characteristics of the end product. 
2.1.2. $\mathrm{Nb}_{2} \mathrm{O}_{5}$ Synthesis Supported on MCM-41 through the Incipient Humidity Impregnation Method (Nb/MC-41-ImpHum)

Impregnation was carried out using $10 \mathrm{~mL}$ of an aqueous solution of niobium oxalate in $0.1 \mathrm{M}$ of oxalic acid with which $1.1034 \mathrm{~g}$ of the MCM-41 material were impregnated, drop by drop, until the whole solid was humid; the amount of niobium oxolate aggregated was $0.4756,0.9512$, and $1.4268 \mathrm{~g}$ to obtain niobium percentages of $10 \%, 20 \%$, and $30 \%$, respectively, in the mesoporous silica material. The humid solid was air dried at $60{ }^{\circ} \mathrm{C}$ and, thereafter, calcined at $550{ }^{\circ} \mathrm{C}$ for $6 \mathrm{~h}$.

\subsection{Characterization of Photocatalysts}

The synthesized solids were micro-structurally characterized using: X-ray diffraction (XRD), Fourier Transformed Infrared Spectroscopy (FTIR), Raman spectroscopy, and transmission electron microscopy (TEM). To perform the IR spectroscopy analysis, the solid was homogenized with spectroscopic grade potassium bromide (99\%, Fischer, Pittsburgh, USA) in an agate mortar. The mixture was subjected to pressure through a 318 stainless steel die until forming a translucent pellet. The sample was analyzed with a Nicolet IR-200 infrared spectrophotometer equipped with EZOMINIC 32 software. A total of 32 scans were conducted at a resolution of $16 \mathrm{~cm}^{-1} / \mathrm{s}$. For the XRD study of the powder samples, a PANalytical X'Pert Pro X-ray diffractometer was used with Bragg Brentano geometry, equipped with $\mathrm{CuK} \alpha(\lambda=1.5406 \AA)$ radiation source, operating with $45 \mathrm{~mA}$ current and voltage of $45 \mathrm{kV}$. Powder $\mathrm{X}$-ray diffraction patterns (PXRD) were registered within the 1 to $10^{\circ}$ interval, as well as between $10^{\circ}$ and $70^{\circ}$, in $2 \theta$, at $0.5^{\circ} / \mathrm{min}$ scan rate. The samples were observed with transmission electron microscopy (Jeol 1200 EX, JEOL, Pleasanton, CA, USA) with an electron beam electric potential acceleration of $80 \mathrm{kV}$. Raman spectra were obtained through EZRaman-N and ProRaman-L-905 (Enwave Optronics, Irvine, USA) Raman analyzers coupled to a Leica DM300 microscope (with a Leica objective that has a magnification/numerical aperture ratio of 40×/0.65), using excitation laser sources of $905 \mathrm{~nm}$ (maximum power $\sim 400 \mathrm{~mW}$ ). The adsorption/desorption of nitrogen was carried out at $-196{ }^{\circ} \mathrm{C}$ in a Micromeritics ASAP 2010 (micromeritics, USA). The samples were previously treated under high vacuum at $150{ }^{\circ} \mathrm{C}$ for $12 \mathrm{~h}$. Finally, elemental composition was determined by SEM-EDX in a Stereoscan 440 Leica microscope (Leica Microsystems, Mannheim, Germany) equipped with an energy dispersive X-ray (EDX) elemental analysis system.

\subsection{Photocatalytic Activities}

\subsubsection{Adsorption Kinetics}

To determine the characteristics of the dye adsorption equilibrium on the molecular sieves, a study was conducted on their adsorption kinetics. For this purpose, $10 \mathrm{mg}$ were taken of each of the solids synthesized and these were dispersed in $200 \mathrm{~mL}$ of methylene blue solution with a concentration of $50 \mathrm{ppm}$, at room temperature, under continuous agitation and complete darkness. Thereafter, every $10 \mathrm{~min}$, aliquots of this suspension were extracted and their spectra were taken, considering the maximum absorbance of the methylene blue at $665 \mathrm{~nm}$ using a UV-Vis spectrophotometer (Spectronic Genesys 6); this procedure was repeated over a total time of $60 \mathrm{~min}$. Then, through mathematical 
calculations and using the calibration curve method, the quantity in milligrams of methylene blue milligrams adsorbed on the solids was determined; the curve for the " $q$ " coefficient (mg of methylene blue adsorbed $/ \mathrm{mg}$ of the photocatalyst) as a function of time was obtained. This permitted determining the time interval the system should be kept in continuous agitation prior to turning on the UV lamps to determine the photodegradation capacity of suspended Nb/MCM-41 solids.

\subsubsection{Photodegradation Effect}

To establish the photodegradation capacity of the synthesized solids, methylene blue powder $(\mathrm{C} 16 \mathrm{H} 18 \mathrm{~N} 3 \mathrm{Cl} \mathrm{S})$ was taken as the reference organic molecule to be degraded. This powder was dissolved in distilled water at a concentration of $55 \mathrm{ppm}$ and $200 \mathrm{~mL}$ were taken from the resulting solution and poured into a $250-\mathrm{mL}$ precipitation glass. Then, $10 \mathrm{mg}$ of the synthesized powders were weighed and added to the methylene blue solution. The mixture was agitated for the time determined from the adsorption kinetics to eliminate or reduce as much as possible the adsorption effect, and then transferred to a "solar simulator" constructed in the laboratory. The suspension was placed in the simulator and the UV lamps (Phillips TUV $15 \mathrm{~W}$ lamps, maximum intensity at $254 \mathrm{~nm}$, Phillips, Lausanne, Switzerland) turned on to begin the photodegradation process. During this process, the system was maintained at a continuous agitation of $500 \mathrm{rpm} / \mathrm{min}$, taking samples periodically every $5 \mathrm{~min}$. The samples were placed in a small quartz container and placed in the UV-Vis spectrophotometer to obtain the system's absorbance value at a wavelength of $665-\mathrm{nm}$; identical steps were followed for the photolysis test, but using only the methylene blue solution. Upon determining the absorbance, the suspension was again poured into the precipitation glass. This process was repeated every 5 min, until minute 20, and then every $10 \mathrm{~min}$ until minute 60 . Finally, the methylene blue percentage degradation over time curve was obtained with respect to the calibration curve. The photodegradation effect was determined by employing a laboratory designed photo-reactor. Monitoring of the disappearance of methylene blue in the solution was carried out using UV-Vis spectroscopy.

\section{Results and Discussion}

\subsection{Characterization of Solids}

The FTIR spectrum of Nb-MCM-41 materials is shown in Figure 1, where bands are observed between 1400 and $400 \mathrm{~cm}^{-1}$ due to the fundamental vibrations of the mesoporous structure. A large band around $3450 \mathrm{~cm}^{-1}$ and a band at $\sim 1630 \mathrm{~cm}^{-1}$ correspond to $\mathrm{O}-\mathrm{H}$ stretching and surface water [24]. The bands at $\sim 1230, \sim 1080$, and $\sim 810 \mathrm{~cm}^{-1}$ are assigned to $\mathrm{Si}-\mathrm{O}$ symmetric and asymmetric stretch vibrations; the band at $\sim 460 \mathrm{~cm}^{-1}$ is characteristic of silica compounds and corresponds to O-Si-O group stretching [25]. This infrared spectrum was the same independent of the procurement method and percent incorporation of niobium.

Raman spectroscopy characterization permits to determine, to a certain degree, the incorporation of niobium pentoxide onto the MCM-41 mesoporous structure. The lack of a neat Raman band in the spectrum at $\sim 680 \mathrm{~cm}^{-1}$ —corresponding to niobium oxide polyhedra symmetric stretching modes—or bands between 200 and $300 \mathrm{~cm}^{-1}$ — assigned to flexion modes of $\mathrm{Nb}-\mathrm{O}-\mathrm{Nb}$ bonds [26] — would indicate a lack of $\mathrm{Nb}_{2} \mathrm{O}_{5}$ crystalline nanoparticles on the silica structure. Bearing this in mind, it can be observed 
in Figure 2 that the Raman band at $\sim 680 \mathrm{~cm}^{-1}$ is present in most of the solids analyzed, except for the mesoporous solids with $10 \%$ niobium synthesized through the sol-gel method, Figure 2 d, and with $20 \%$ niobium obtained through the incipient impregnation method, Figure $2 b$, which indicates that most of the niobium added was incorporated into the silica structure [26]. However, in the other solids the presence of this band indicates that not all the niobium added was incorporated into the silica, but that a certain percentage of it was leached. Though the increase occurring in the background of the Raman spectra, Figure 2b,d, can be associated with the fluorescence of organics present in the sample [27], due to the presence of carbon in the sample as it was found in the images of SEM-EDS (Figure 3), however the carbon content is small.

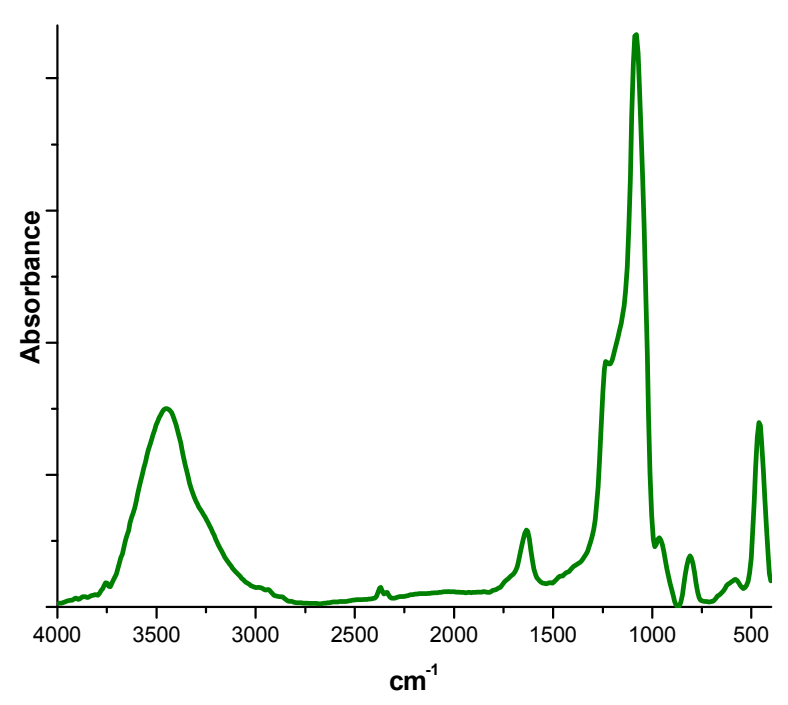

Figure 1. Infrared spectrum of Nb-MCM-41 sample.
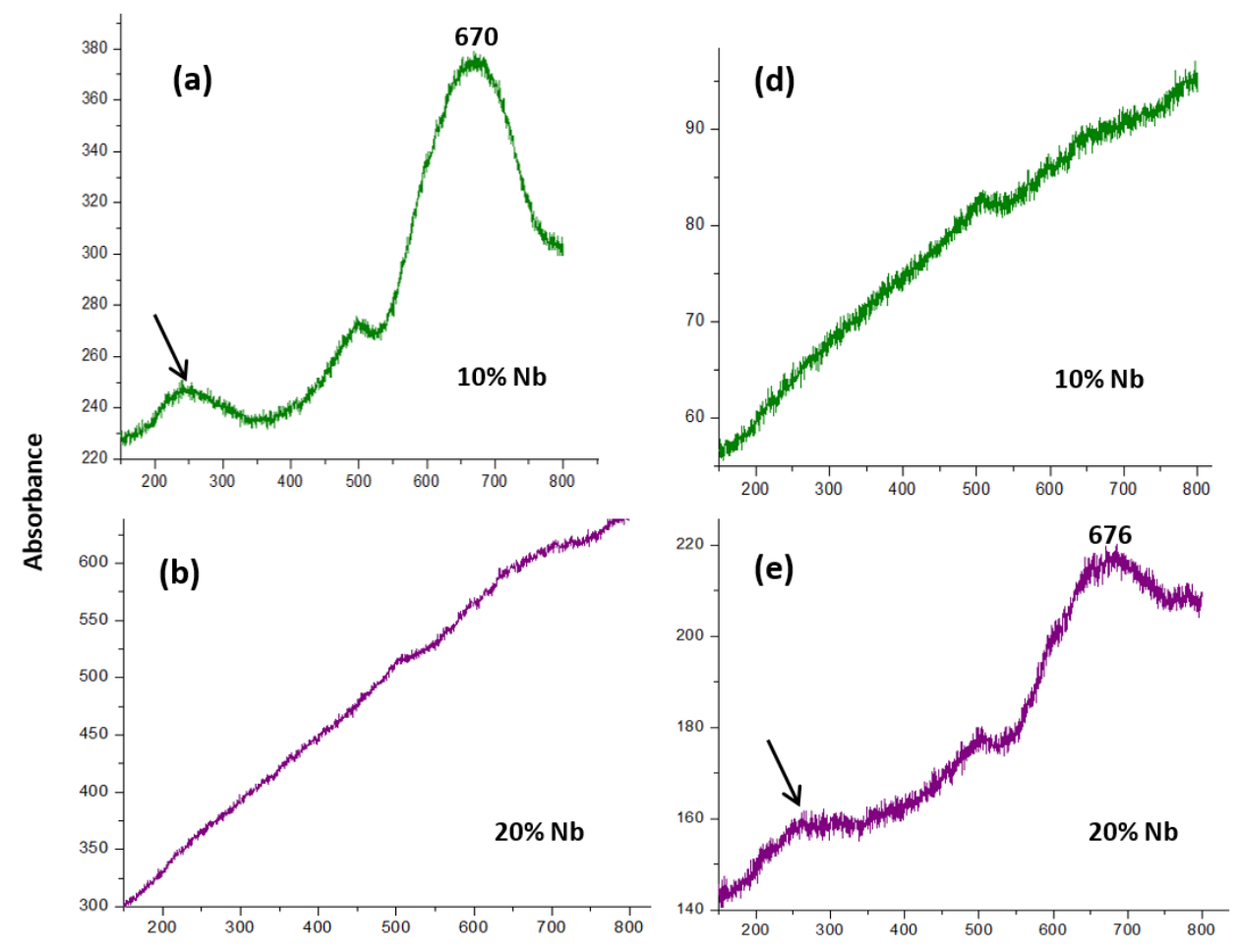

Figure 2. Cont. 


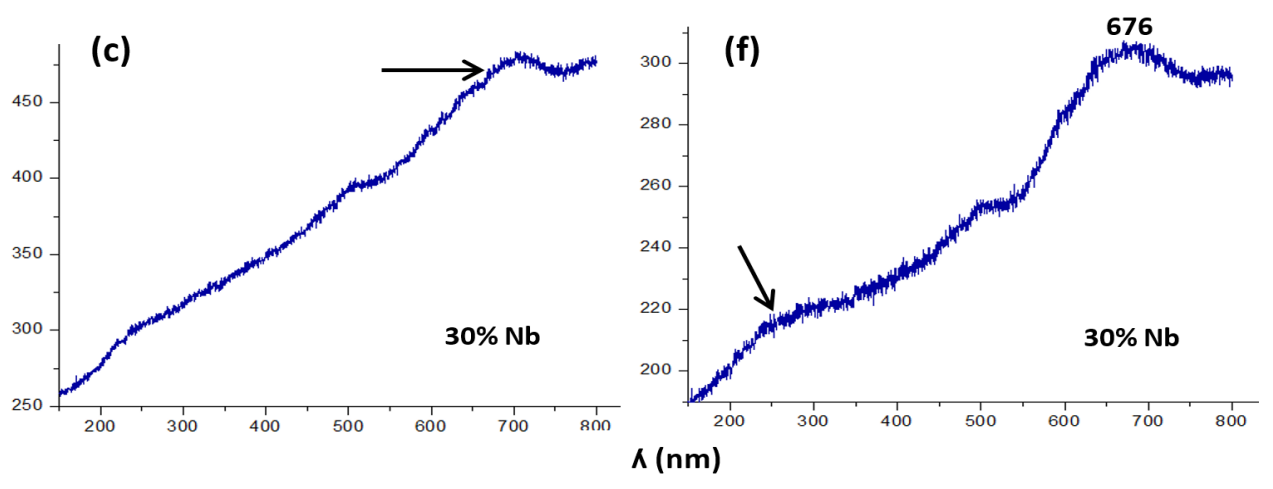

Figure 2. Raman spectrum of Nb-MCM-41 through the incipient impregnation method (a), (b), and (c), and through the sol-gel method (d), (e), and (f).

In order to verify the niobium concentration on the surface of the MCM-41 mesoporous structure, EM-EDS analysis was conducted, shown in Figure 3.
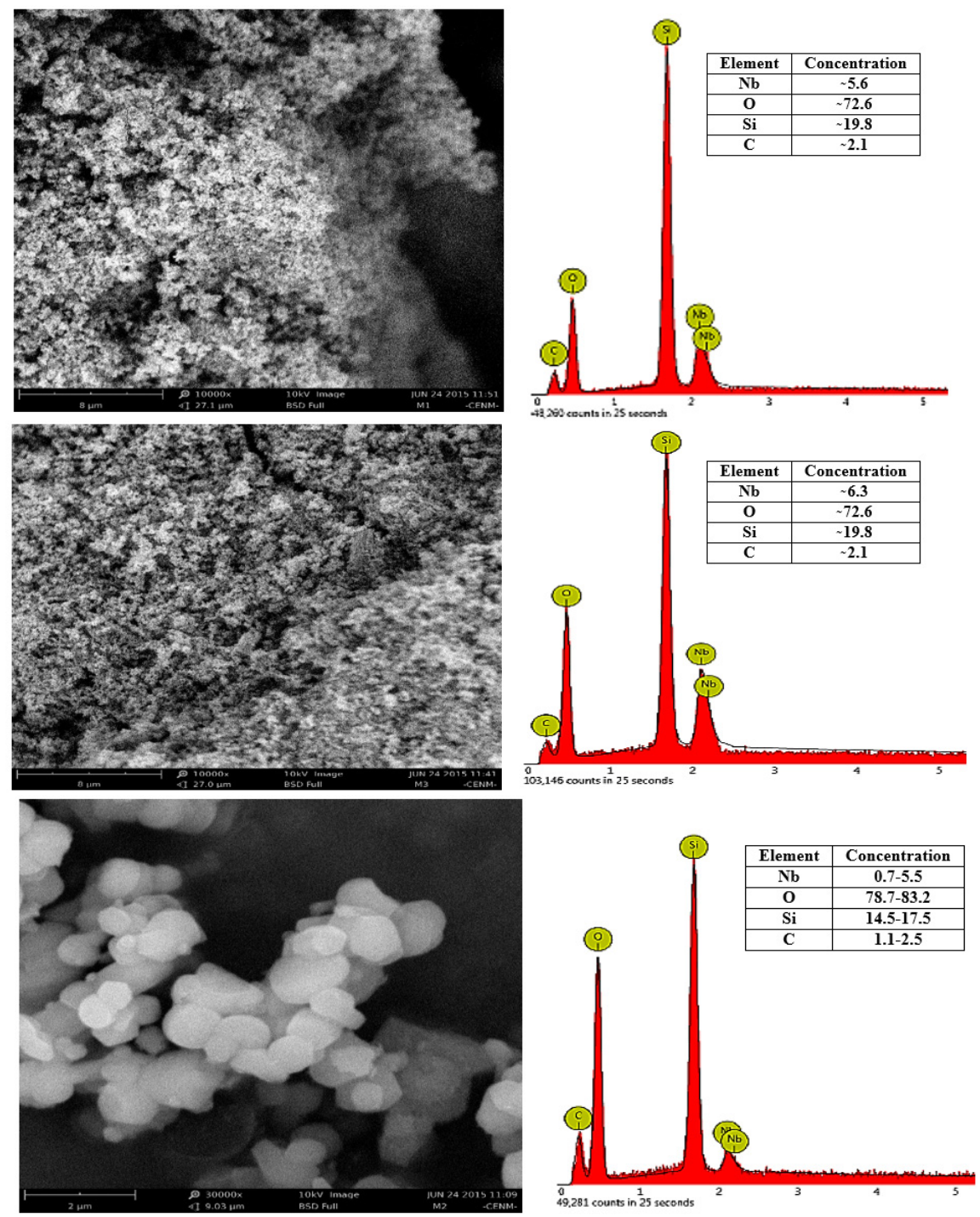

Figure 3. SEM-EDS photograph of Nb-MCM-41 (a) 20\%-Nb-MCM-41, (b) 30\%-Nb-MCM-41 powders obtained through the sol-gel method and (c) 20\%-Nb-MCM-41 powder obtained through the incipient impregnation method. 
The Nb-MCM-41 powders obtained through the sol-gel method showed a greater distribution of niobium on the surface. In those obtained by the incipient impregnation method, however, the niobium distribution was not homogeneous.

The Nb-MCM-41 materials obtained using the incipient impregnation method (Figure 4) produces an X-ray diffractogram characteristic of MCM-41, which contains four peaks at low angles, as noted in the figure; the first of these is the most intense peak, appearing around $2 \theta=2^{\circ}$, corresponding to Miller index (100). Other lower-intensity reflections appear between $3^{\circ}<2 \theta<10^{\circ}$, corresponding to Miller indexes (110) and (200), which verifies a hexagonal symmetry of the structure [24,25,27]; the X-ray diffractogram is the same independent of the percentage of niobium incorporated. This result is similar to that obtained by Ziolek and Nowak [16].

Furthermore, observing the complete diffractogram between $10^{\circ}$ and $90^{\circ}$, Figure $4 \mathrm{~b}$, similar for all samples impregnated with $\mathrm{Nb}$, two ridges are observed in the regions where, normally, the peaks characteristic of $\mathrm{Nb}_{2} \mathrm{O}_{5}$ are located, so that these samples may contain crystals of this oxide forming. Regarding the solids obtained using the sol-gel method, these produce a diffractogram (Figure 5) that differs from the typical MCM-41structure, given that it does not show this structure's characteristic peaks. However, these solids show peaks at low angles, which could indicate that a mesoporous structure is being obtained, but not an MCM-41 type structure.

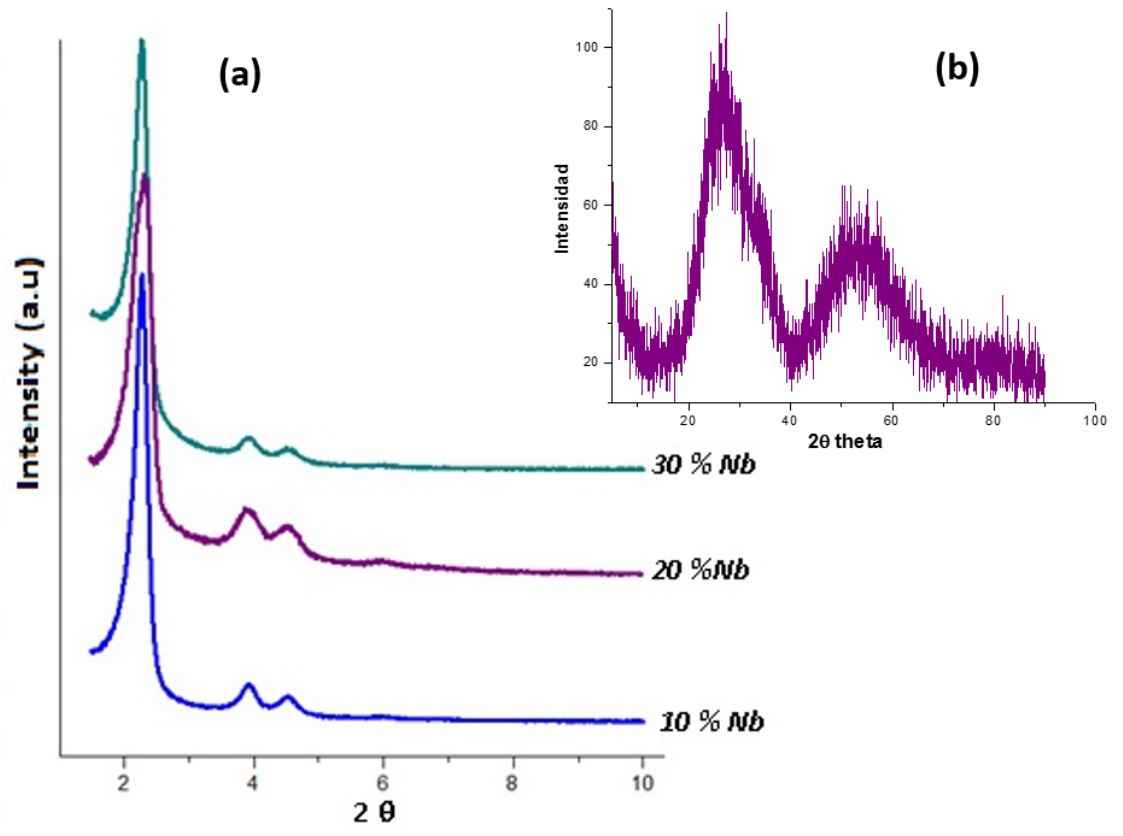

Figure 4. X-ray diffractogram of Nb-MCM-41 obtained using the incipient impregnation method to (a) in the region of low angles, and (b) in the high-angle region.

These diffraction patterns are different from those obtained by Ziolek and Nowak [16] in that the mesoporous Nb-MCM-41 synthesized by the sol-gel method should show a different pore structure. Given the similarity of the XRD patterns of Figure 5 to the small-angle XRD patterns of the mesoporous $\mathrm{Nb}$ samples synthesized by Chen et al. [19] that showed obvious peaks between 0.58 and 1.58 and no other peak at higher degrees, it can be concluded that these came from a less ordered mesoporous structure and that the $d$ value of each mesoporous sample would lie between 7.7 and $14.9 \mathrm{~nm}$. 
Moreover, observing the full diffractogram between $10^{\circ}$ and $90^{\circ}$, Figure $5 \mathrm{~b}$, similar for all the samples synthesized using different concentrations of $\mathrm{Nb}$ precursor, this is similar to that of Figure $4 \mathrm{~b}$, so that it is possible these samples also contain in their structure some forming $\mathrm{Nb}_{2} \mathrm{O}_{5}$ crystals.

To corroborate the mesoporous structure, the $\mathrm{N}_{2}$ adsorption and desorption isotherms are shown in Figure 6. This analysis allowed to determine of the Nb-containing mesoporous MCM-41 solid along with large surface areas after the incorporation of niobium to the structure of the silica. As the load increases niobium, surface area decreases as expected (Table 1).

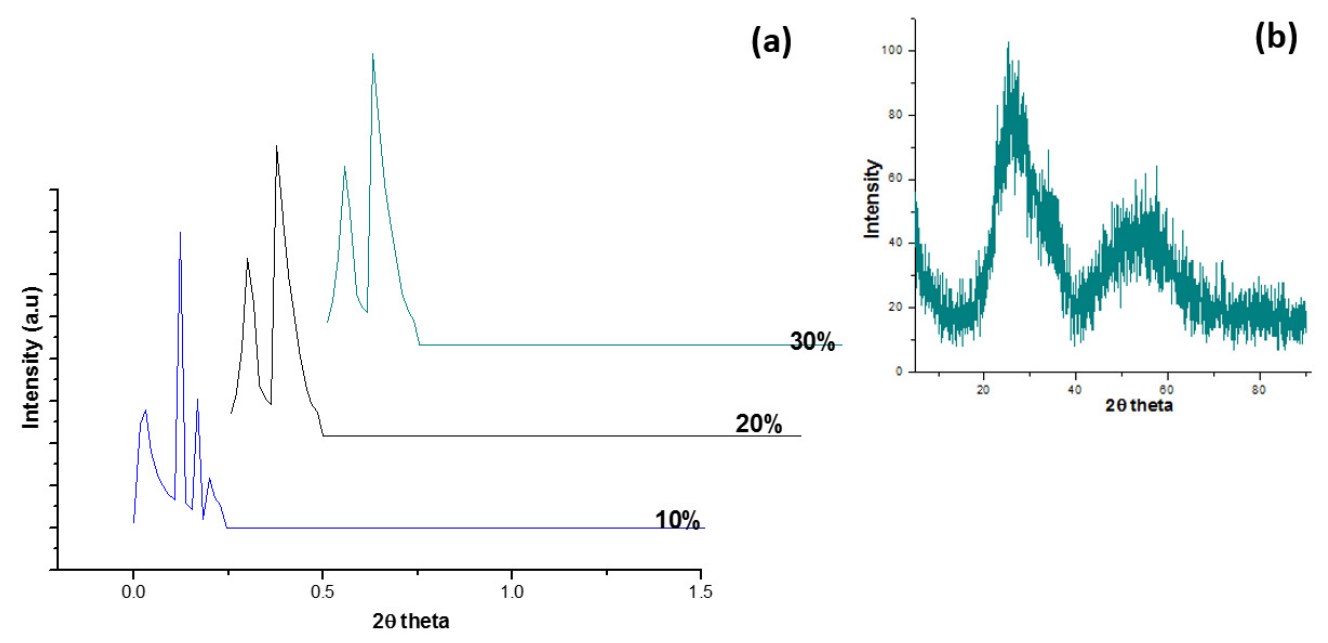

Figure 5. X-ray diffractogram of Nb-MCM-41 obtained through the sol-gel method with $10 \%, 20 \%$ and $30 \%$ of niobium.
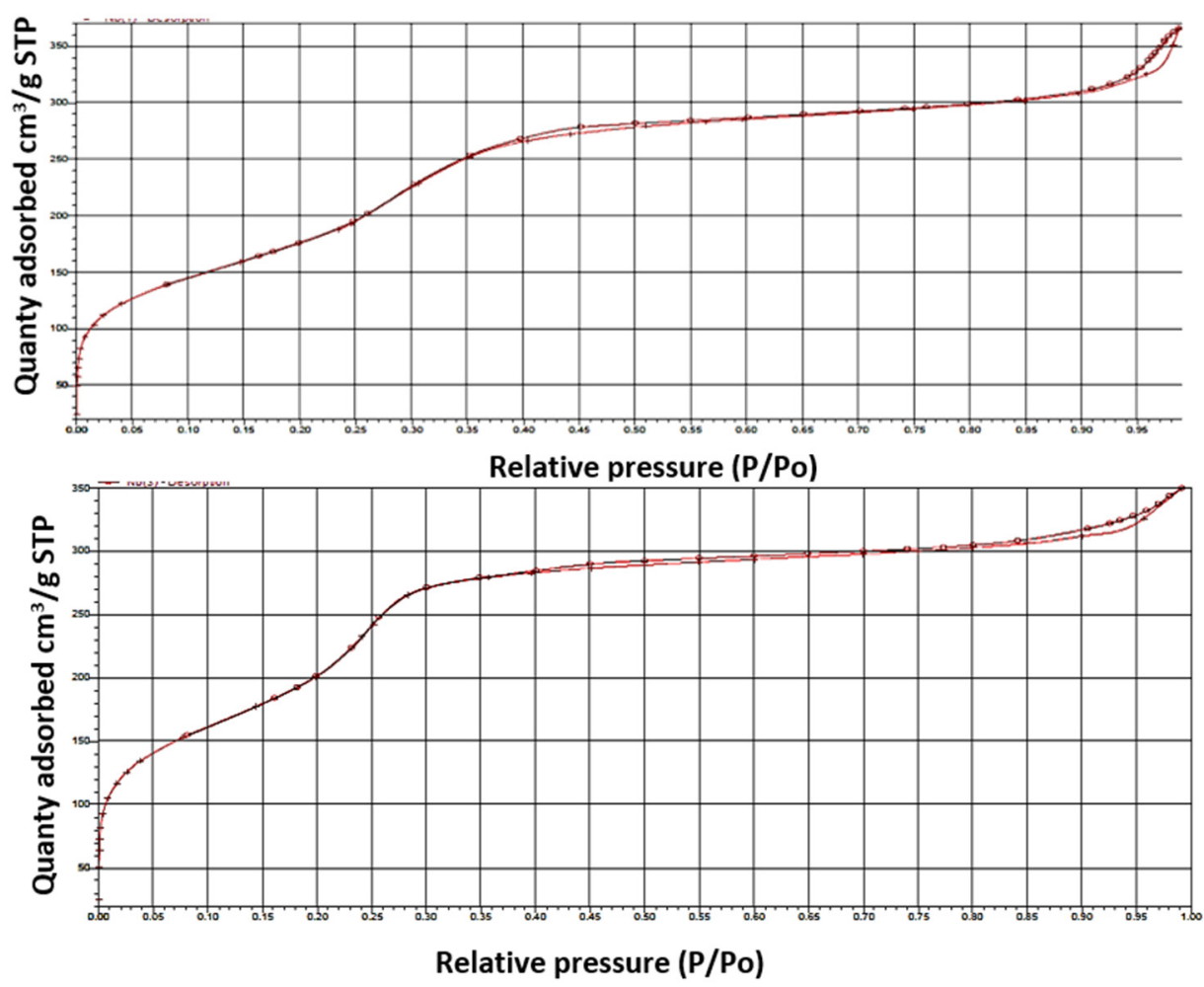

Figure 6. $\mathrm{N}_{2}$ adsorption and desorption isotherms of $20 \% \mathrm{Nb}-\mathrm{MCM}-41$ obtained through (a) sol-gel and (b) incipient impregnation methods, respectively. 
However, although the samples exhibited a mesoporous phase, which was corroborated using DRX at low angles, their hysteresis loop is different compared to that of MCM-41, possibly through the formation of species on the surface of the pores from the niobium, which leads to a "plugging" of the channels, and which was reflected in the narrowness of the hysteresis loop area [28], as well as decreasing both the surface area and pore size. This plugging would be generated by the synthesis methods used to obtain the samples of interest. In the case of impregnation of the MCM-41, it is very likely that niobium species are deposited, during the impregnation process, in the pores of the substrate (MCM-41), plugging them and causing a reduction in the hysteresis loop (see Figure 6b). In the case of the samples synthesized by sol-gel, after forming the micelles and adding the organic $\mathrm{Nb}$ precursor (ammonium oxalate), it is possible that due to its nature, some of it is distributed within the micelles (lyophobic area) and remains there throughout the process of synthesis, leading finally to plugging of the pores and thus a reduction of the hysteresis loop (Figure 6a). Clearly, on increasing the concentration of $\mathrm{Nb}$ precursor, a greater plugging of the pores can be expected, and a reduction in their size, as shown in Table 1.

Table 1. Textural properties of mesoporous solids synthesized in this work.

\begin{tabular}{ccccccc}
\hline Sample & Mass (g) & $\begin{array}{c}\text { BET Surface } \\
\text { Area }\left(\mathbf{m}^{2} / \mathbf{g}\right)\end{array}$ & $\begin{array}{c}\text { Correlation } \\
\text { Coefficient }\end{array}$ & $\begin{array}{c}\text { Pore Volume } \\
\left(\mathbf{c m}^{3} / \mathbf{g}\right)\end{array}$ & $\begin{array}{c}\text { Pore Size } \\
(\mathbf{n m})\end{array}$ & $\begin{array}{c}\text { Pore } \\
\text { Type }\end{array}$ \\
\hline $\begin{array}{c}\text { 20\% Nb-MCM-41 } \\
\text { sol-gel method }\end{array}$ & 0.3896 & $\begin{array}{c}684.1391 \\
\pm 24.5850\end{array}$ & 0.9967814 & 0.618766 & 2.61 & Mesopore \\
$\begin{array}{c}\text { 30\% Nb-MCM-41 } \\
\text { sol-gel method }\end{array}$ & 0.0659 & $\begin{array}{c}496.8287 \\
\pm 10.1249\end{array}$ & 0.9993733 & 0.458849 & 2.42 & Mesopore \\
$\begin{array}{c}\text { 20\% Nb-MCM-41 } \\
\text { incipient impregnation } \\
\text { method }\end{array}$ & 0.0441 & $\begin{array}{c}836.4673 \\
\pm 41.6990\end{array}$ & 0.9924810 & 0.610336 & 2.44 & Mesopore \\
\hline
\end{tabular}

The TEM micrographs for the Nb-MCM-41 solids obtained through the incipient impregnation method (Figure 7) should not present optimal pore distribution, mainly because these solids were calcined again following impregnation with the niobium precursor, which would generate some loss of organized structure.

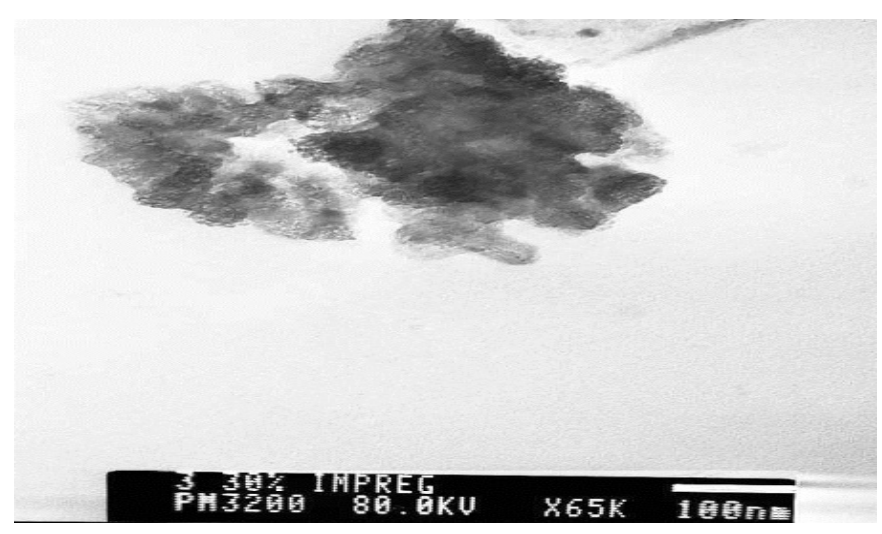

Figure 7. TEM micrographs corresponding to Nb-MCM-41 mesoporous solids obtained using the incipient impregnation method with $30 \%$ niobium. 
The TEM micrographs corresponding to Nb-MCM-41 mesoporous solids synthesized using the sol-gel method are also shown in Figure 8, illustrating that pore distribution is uniform, which indicates that the pore size for these solids ranges between 2 and $10 \mathrm{~nm}$. These transmission electron micrographs are typical of mesoporous structures, according to several reports [29].

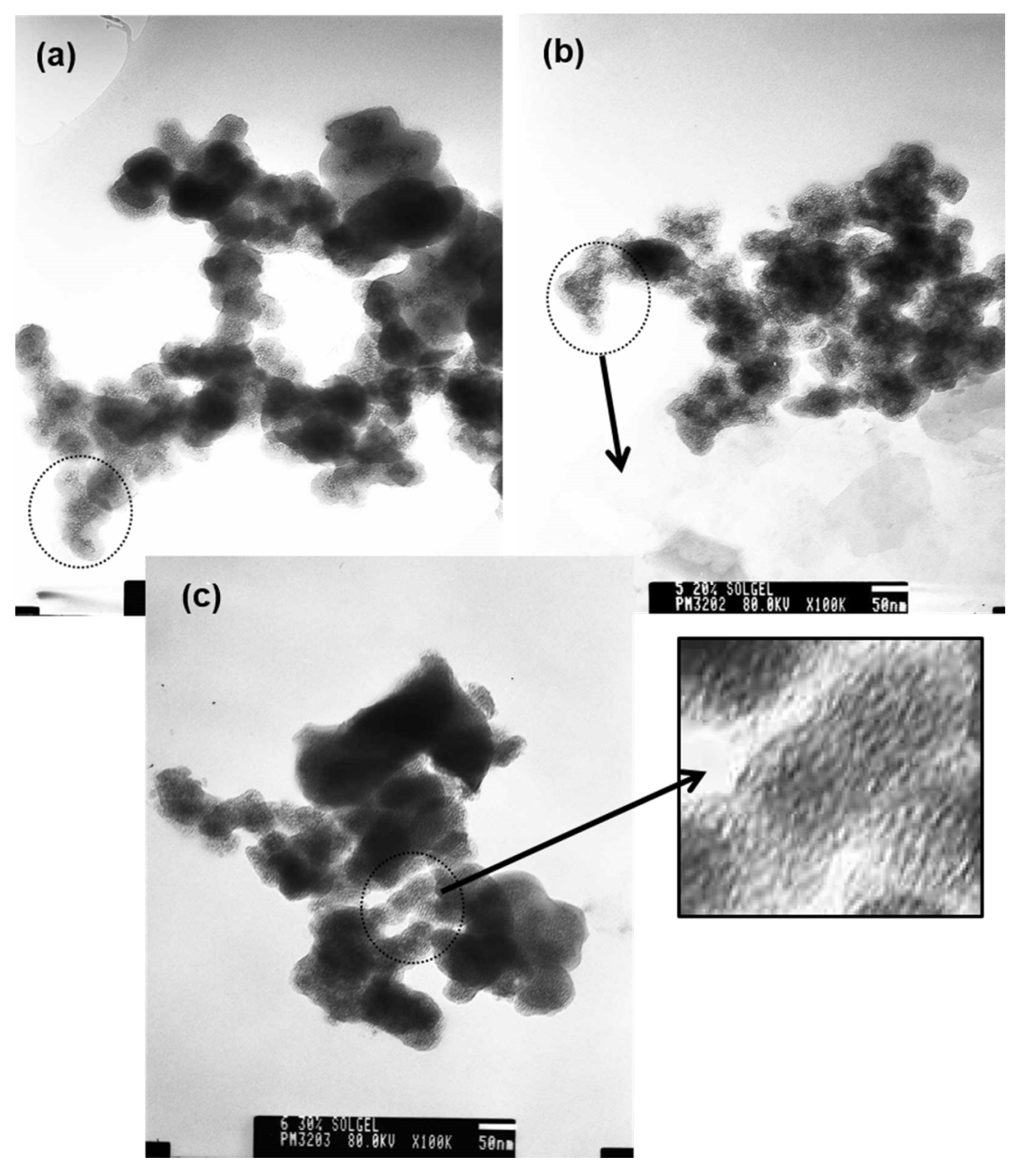

Figure 8. TEM micrographs corresponding to Nb-MCM-41 mesoporous solids obtained using the sol-gel method with niobium percentages of (a) 10\%, (b) 20\%, and (c) 30\%.

\subsection{Adsorption Kinetics and Results of Photodegradation Effect}

The methylene blue adsorption kinetics corresponding to mesoporous solids are shown in Figure 9. These curves show marked differences in dye adsorption rates obtained by the solids, depending on the methodology used in the incorporation of niobium, as well as on the percentage of this cation on the mesoporous silica MCM-41. The solids with the highest dye adsorption capacity were those containing $10 \% \mathrm{Nb}$, obtained through the sol-gel method, and $20 \% \mathrm{Nb}$ obtained using the incipient impregnation method. This result indicates that greater uniformity of niobium distribution on the surface of the mesoporous solid guarantees higher adsorption efficiency, as indicated by the Raman spectra (Figure 2).

Further, solids with higher adsorption capacity did not show leaching or formation of $\mathrm{Nb}_{2} \mathrm{O}_{5}$ clusters. What is evident in the adsorption kinetics (Figure 9) is that the $\mathrm{Nb}$ percentage does not notably affect dye adsorption. 

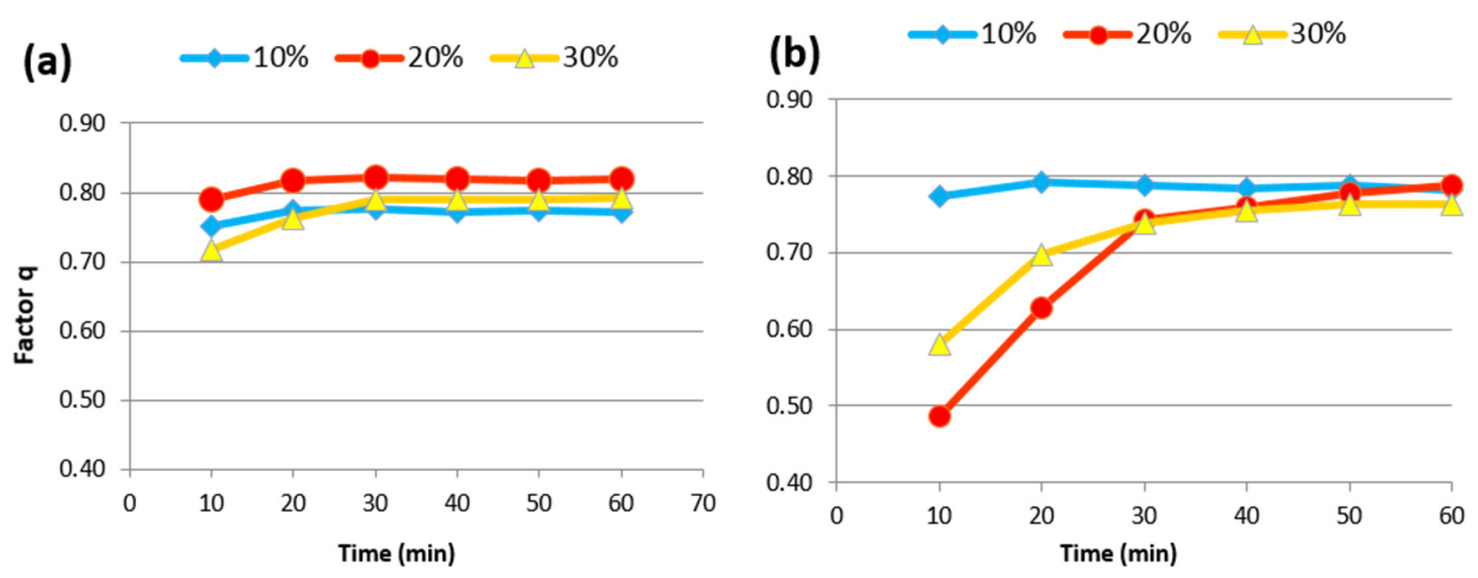

Figure 9. Adsorption kinetics of Nb-MCM-41 mesoporous solids obtained using the sol-gel (a) and impregnation method (b) (Factor $q$ : $\mathrm{mg}$ methylene blue adsorbed/mg photocatalyst).

Although all the solids exhibit nearly the same total adsorption capacity, the behavior of the curves, as shown in Figure 9, is different, leading to the conclusion that the adsorption mechanisms were different. This could be due, in the main, to the differences in the microstructure of the samples, since as seen in the XRD at low angles, they clearly show a different mesophase, which could be closely related to the diffusion of the reagents. It is necessary, in future, to carry out a careful study to learn more about the mechanisms of adsorption as regards these samples and relate them to their characteristics (Table 1).

The process of photodegradation of methylene blue brought about by the Nb/MCM-41 mesoporous solids synthesized in this work is illustrated in Figures 10 and 11. The results show that for both methods of $\mathrm{Nb}$ incorporation, solids with $20 \% \mathrm{Nb}$ showed a better photodegradation response. By comparing the curves, it is evident that the solid obtained using the sol-gel method showed a much higher degradation percentage than the solid obtained using incipient impregnation, with comparative figures of $60.6 \%$ and $16.7 \%$, respectively (Figures 10 and 11 ).

Additionally, considering these results, and taking into account those of the Raman spectroscopy (Figure 2), it can be concluded that better niobium dispersion on the silica leads to a reduced photodegradation effect. In other words, although the uniform incorporation of niobium on mesoporous silica favored adsorption capacity, this condition notably diminished the photodegradation effect. This is not the case in the samples containing $10 \% \mathrm{Nb}$, where the reverse is true, i.e., better niobium dispersion over the mesoporous silica indicates a greater photodegradation effect. This shows that it is necessary to conduct more systematic studies on both the niobium percentages in the sample and the uniformity of niobium dispersion, in the context of the effect of these on photodegradation capacity. Despite the fact that the $30 \% \mathrm{Nb}-\mathrm{MCM}-41$ sample contains more niobium, as was determined in SEM-EDS, it may be that some of the $\mathrm{Nb}$ is not available to take part in photocatalysis, for example the $\mathrm{Nb}$ can be deposited in the zeolite porosities. Furthermore, as the amount of niobium in photocatalysts is increased, the surface area decreases, which indicates that there are fewer active sites available.

What is indeed evident in the results obtained is that the solids synthesized using the sol-gel method presented a greater photodegradation response than those obtained using the incipient impregnation method (Figures 10 and 11). This behavior could be justified considering that the pores observed in the TEM micrographs (Figure 8) of solids from the sol-gel method are more uniform than those observed in 
the incipient impregnation samples. This characteristic would provide a bigger surface area in the former samples, improving the photocatalytic process.

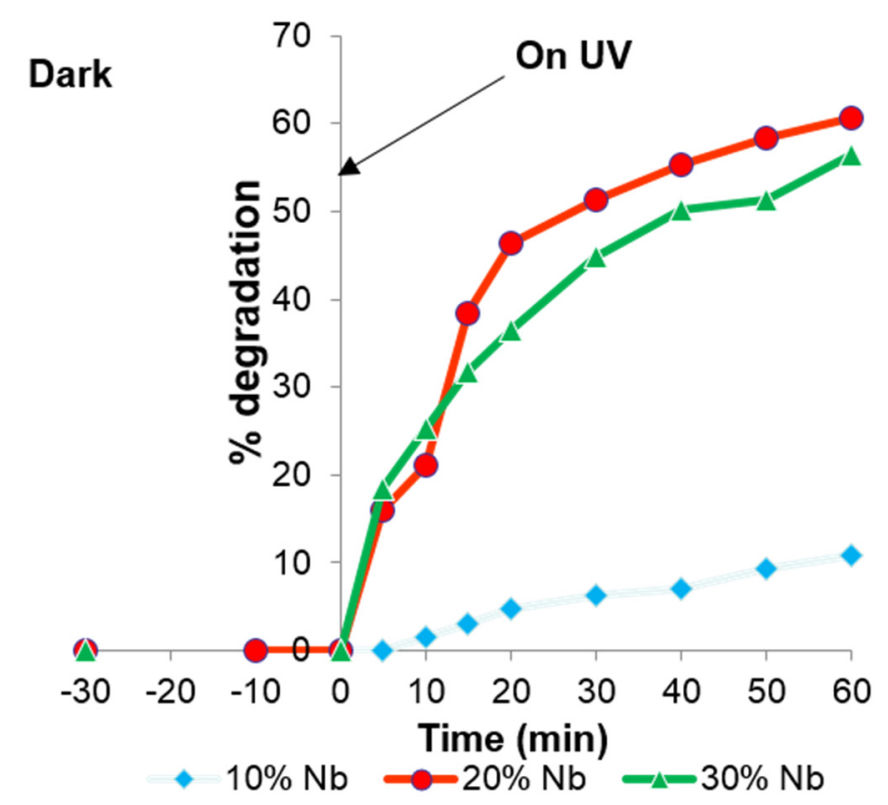

Figure 10. Degradation percentage of methylene blue, in function of irradiation time (sol-gel method).

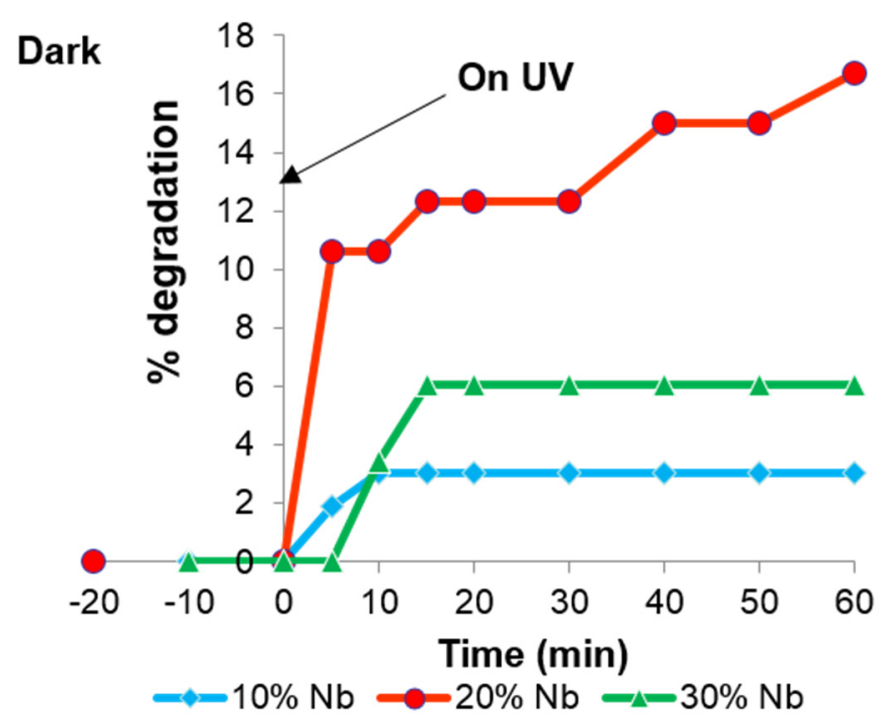

Figure 11. Degradation percentage of methylene blue vs. irradiation time (incipient impregnation method).

The photocatalysts studied in this work can be considered, mainly those obtained by impregnation as a result of their behavior and the results obtained, as another type of photocatalyst, i.e., "single-site photocatalyst". In general, this single-site photocatalysis contains isolated polyhedral coordination transition metal oxides such as the oxides of $\mathrm{Ti}^{4+}, \mathrm{V}^{5+}, \mathrm{Cr}^{6+}$ and $\mathrm{Mo}^{6+}$ as active sites [30]; in this study, $\mathrm{Nb}^{5+}$ oxide. These species were designed on a mesoporous molecular MCM-41 surface and were considered to be highly dispersed at the atomic level on its surface (mainly in the samples obtained by 
impregnation) or within the frameworks and to be well-defined active sites. These local structures of our single-site $\mathrm{Nb}$ photocatalysts could explain the results obtained using Raman spectroscopy (Figure 2) and the behaviour of photocatalysts in the reduction of methylene blue (Figures 10 and 11). The photocatalytic properties of single-site photocatalysts Nb-MCM-41 could be attributed to the ligand to metal charge transfer (LMCT) process of the isolated niobium oxide with tetrahedral coordination. Under light irradiation, the charge transfer excited triplet state of niobium oxide was formed and electron-hole pairs were localized in close proximity to its photo-excited states [30]. Single-site photocatalysts exhibit unique and fascinating photocatalytic performances based on the reactions of specific photo-excited species. This process cannot be carried out on bulk photocatalysts [30]. Finally, the method of synthesis is clearly important in obtaining the single-site photocatalyst Nb-MCM-41 and for its functionality.

\section{Conclusions}

Regarding Nb-MCM-41 synthesis, the solids synthesized using incipient impregnation show a hexagonally ordered MCM-41 type mesoporous structure, as observed in the X-ray diffractograms. Solids obtained by the sol-gel method, meanwhile, did not present this type of structure. However, the mesoporosity of the systems is evident in the transmission electron micrographs that indicate a mesoporous-type ordered structure, although the family to which they correspond would still need to be determined.

Using incipient impregnation, better dispersion was observed on the silica, given that for up to $20 \%$ of incorporation, no $\mathrm{Nb}_{2} \mathrm{O}_{5}$ leaching was observed. This method thus shows greater advantages for niobium incorporation in silica. As such, greater dispersion appears to generate a less homogeneous pore size and a reduced photodegradation effect.

The Nb-MCM-41 mesoporous solids synthesized using the sol-gel method gave a greater photodegradation response than those synthesized through the incipient impregnation method. The highest effectiveness in photodegradation of the methylene blue molecule was obtained with the mesoporous solid synthesized through the sol-gel method, at a level of $20 \% \mathrm{Nb}$. This suggests that it is important to determine the structure obtained through the sol-gel method, to understand better the interaction between silica and niobium and the resulting effect on photodegradation. The results obtained in this study reiterate the importance of the method of synthesis used to obtain the mesoporous materials on their functionality.

\section{Acknowledgements}

The authors would like to thank CBMM for providing the niobium metal precursor. We are very grateful to the University of Cauca for offering the use of its laboratory facilities and making the necessary research time available. We are also grateful to Colin McLachlan for suggestions relating to the English text. This work was supported by project ID 4032 - VRI of the University of Cauca.

\section{Author Contributions}

C.D.G and J.E.R both coordinated and supervised the different research projects involving synthesis and photocatalytic applications; C.D.G prepared the manuscript. All authors read and approved the manuscript. 


\section{Conflicts of Interest}

The authors declare no conflict of interest.

\section{References}

1. Gaya, U.I. Heterogeneous Photocatalysis Using Inorganic Semiconductor Solids; Springer Science + Business Media: Dordrecht, The Netherlands, 2014.

2. Kaneko, M.; Okura, I. Photocatalysis: Science and Technology; Kodansha-Springer: New York, NY, USA, 2002.

3. Hoffmann, M.R.; Martin, S.T.; Choi, W.; Bahnemann, D.W. Environmental applications of semiconductor photocatalysis. Chem. Rev. 1995, 95, 69-96.

4. Coronado, J.M.; Fresno, F.; Hernández-Alonso, M.D.; Porteña, R. Design of Advanced Photocatalytic Materials for Energy and Environmental Applications; Springer-Verlag: London, UK, 2013.

5. Zhou, B.; Raja, R.; Han, S.; Somorjai, G.A. Nanotechnology in Catalysis; Springer: New York, NY, USA, 2007; Volume 3.

6. Brus, L. Electronic wave functions in semiconductor clusters: experiment and theory. J. Phys. Chem. 1986, 90, 2555-2560.

7. Nozik, A.J.; Williams, F.; Nenadovic, M.T.; Rajh, T.; Micic, O.I. Size quantization in small semiconductor particles. J. Phys. Chem. 1985, 89, 397-399.

8. Stroyuk, O.L.; Kuchmiy, S.Y.; Kryukov, A.I.; Pokhodenko, V.D. Semiconductor Catalysis and Photocatalysis on the Nanoscale; Nova Science Publishers, Inc.: New York, NY, USA, 2010.

9. Shchukin, D.G.; Sviridov, D.V. Photocatalytic processes in spatially confined micro- and nanoreactors. J. Photochem. Photobiol. C 2006, 7, 23-39.

10. Chen, H.; Chen, S.; Quan, X.; Zhang, Y. Structuring a $\mathrm{TiO}_{2}$-based photonic crystal photocatalyst with Schottky junction for efficient photocatalysis. Environ. Sci. Technol. 2010, 44, 451-455.

11. Kim, S.; Kwak, S.Y. Photocatalytic inactivation of E. coli with mesoporous $\mathrm{TiO}_{2}$ coated film using the film adhesion method. Environ. Sci. Technol. 2009, 43, 148-151.

12. Yang, H.-C.; Lin, H.-Y.; Chien, Y.-S.; Wu, J.C.-S. Mesoporous $\mathrm{TiO}_{2} / \mathrm{SBA}_{-15}$ and $\mathrm{Cu} / \mathrm{TiO}_{2} / \mathrm{SBA}-15$ composite photocatalysts for photoreduction of $\mathrm{CO}_{2}$ to methanol. Cat. Lett. 2009, 131, 381-387.

13. Li, X.; Lu, K.; Deng, K.; Tang, J.; Su, R.; Sun, J.; Chen, L. Synthesis and characterization of ZnO and $\mathrm{TiO}_{2}$ hollow spheres with enhanced photoreactivity. Mat. Sci. Eng. B 2009, 158, 40-47.

14. Ji, P.; Zhang, J.; Chen, F.; Anpo, M. Ordered mesoporous $\mathrm{CeO}_{2}$ synthesized by nanocasting from cubic Ia3d mesoporous MCM-48 silica: Formation, characterization and photocatalytic activity. $J$. Phys. Chem. C 2008, 112, 17809-17813.

15. Chen, Y.; Hu, L.; Wang, M.; Min, Y.; Zhang, Y. Self-assembled $\mathrm{Co}_{3} \mathrm{O}_{4}$ porous nanostructures and their purification and their photocatalytic activity. Colloids Surf. A 2009, 336, 64-68.

16. Ziolek, M.; Nowak, I. Synthesis and characterization of niobium-containing MCM-41. Zeolites 1997, 18, 356-360.

17. Ziolek, M.; Sobezak, I.; Nowak, I.; Decyk, P.; Lewandowaska, A.; Kujawa, J. Nb-containing mesoporous molecular sieves-Possible application in the catalytic processes. Micro. Mesop. Mater. 2000, 35-36, 195-207. 
18. Ziolek, M.; Sobezak, I.; Lewandowska, A.; Nowak, I.; Decyk, P.; Renn, M.; Jankowska, B. Oxidative properties of niobium-containing mesoporous silica catalysts. Catal. Today 2001, 70, 169-181.

19. Chen, X.; Yu, T.; Fan, X.; Zhang, H.; Li, Z.; Ye, J.; Zou, Z. Enhanced activity of mesoporous $\mathrm{Nb}_{2} \mathrm{O}_{5}$ for photocatalytic hydrogen production. Appl. Surf. Sci. 2007, 253, 8500-8506.

20. Lin, H.-Y.; Yang, H.-C.; Wang, W.-L. Synthesis of mesoporous $\mathrm{Nb}_{2} \mathrm{O}_{5}$ photocatalysts with Pt, Au, $\mathrm{Cu}$ and $\mathrm{NiO}$ cocatalyst for water splitting. Catal. Today 2011, 174, 106-113.

21. Lopes, O.F.; Paris, E.C.; Ribeiro, C. Synthesis of nanoparticles through the oxidant peroxide method applied to organic pollutant photodegradation: A mechanistic study. Appl. Catal. B Environ. 2014, 144, 800-808.

22. Oliveira, L.C.A.; Ramalho, T.C.; Goncalves, M.; Cereda, F.; Carvalho, K.T.; Nazzarro, M.S.; Sapag, K. Pure niobia as catalyst for the oxidation of organic contaminants: mechanism study via ESI-MS and theoretical calculations. Chem. Phys. Lett. 2007, 446, 133-137.

23. Esteves, A.; Oliveira, L.C.A.; Ramalho, T.C.; Goncalves, M.; Anastacio, A.S.; Carvalho, H.W.P. New materials base on modified synthetic $\mathrm{Nb}_{2} \mathrm{O}_{5}$ as photocatalyst for oxidation of organic contaminants. Catal. Commun. 2008, 10, 330-332.

24. Gallo, J.M.R.; Paulino, I.S.; Schuchardt, Ulf. Cyclooctene epoxidation using Nb-MCM-41 and Ti-MCM-41 synthesized at room temperature. Appl. Catal. A 2004, 266, 223-227.

25. Gallo, J.M.R.; Pastore, H.O.; Schuchardt, U. Silylation of [Nb]-MCM-41 as an efficient tool to improve epoxidation activity and selectivity. J. Catal. 2006, 243, 57-63.

26. Nowak, I.; Misiewicz, M.; Ziolek, M.; Kubacka, A.; Corte's Corberán, V.; Sulikowski, B. Catalytic properties of niobium and gallium oxide systems supported on MCM-41 type materials. Appl. Catal. A 2007, 325, 328-335.

27. Anilkumar, M.; Hölderich, W.F. Highly active and selective Nb modified MCM-41 catalysts for Beckmann rearrangement of cyclohexanone oxime to E-caprolactam. J. Catal. 2008, 260, 17-29.

28. An, X.; Gao, C. Synthesis of mesoporous N-doped $\mathrm{TiO}_{2} / \mathrm{ZnAl}$-layered double oxides nanocomposite for efficient photodegradation of methyl orange. Mater. Sci. Semicond. Process. 2015, 34, 162-169.

29. Solmaza, A.; TimurDogu, S.B. Synthesis and characterization of V, Mo and Nb incorporated micro-mesoporous MCM-41 materials. Mater. Chem. Phys. 2011, 125, 148-155.

30. Kamegawa, T.; Yamashita, H. Solar energy conversion using single-site photocatalysts. In New and Future Developments in Catalysis: Solar Photocatalysis; Suib, L., Ed.; Elsevier: Amsterdan, The Netherlands, 2013; pp. 103-119.

(C) 2015 by the authors; licensee MDPI, Basel, Switzerland. This article is an open access article distributed under the terms and conditions of the Creative Commons Attribution license (http://creativecommons.org/licenses/by/4.0/). 